\title{
Magnetic field in supernova remnant SN 1987A
}

\author{
E.G. Berezhko ${ }^{1}$ L.T. Ksenofontov ${ }^{1}$ \\ berezhko@ikfia.ysn.ru
}

\begin{abstract}
A nonlinear kinetic theory of cosmic ray (CR) acceleration in supernova remnants is employed to investigate the properties of the remnant SN 1987A. It is shown that a large downstream magnetic field $B_{d} \approx 10 \mathrm{mG}$ is required to fit the existing observational data. Such a strong field together with the strong shock modification due to CR backreaction provides the steep and concave radioemission spectrum and considerable synchrotron cooling of high energy electrons which diminish their X-ray synchrotron flux below the observed Chandra flux which has to be considered as an upper limit for nonthermal X-ray emission. The expected $\gamma$-ray energy flux at TeV-energies at the current epoch is $2 \times 10^{-13} \mathrm{erg} /\left(\mathrm{cm}^{2} \mathrm{~s}\right)$.
\end{abstract}

Subject headings: (ISM:) cosmic rays - acceleration of particles - shock waves (stars:) supernovae: individual (SN 1987A) - radiation mechanisms: non-thermal - X-rays: individual (SN 1987A)

\section{Introduction}

Supernova (SN) 1987A occurred in the Large Magellanic Cloud. It has been extensively studied in all wavelengths from radio to $\gamma$-ray. The initial short outburst of radio emission (Turtle et al. 1987) is attributed to the synchrotron emission of electrons accelerated by the SN shock propagated in the free wind of presupernova star, which was the blue supergiant (BSG) (Chevalier \& Fransson 1987). After about 3 years radio emission was detected again (Staveley-Smith et al. 1992; Gaensler et al. 1997) as well as the monotonically increased Xray emission (Gorenstein et al. 1994; Hasinger et al. 1996). This second increase of emission is attributed to the entrance of the outer SN shock into the thermalized BSG wind and then in the H II region occupied by much more dense matter, consists of the swept up wind of red supergiant (RSG) progenitor star (Chevalier \& Dwarkadas 1995).

\footnotetext{
${ }^{1}$ Yu.G. Shafer Institute of Cosmophysical Research and Aeronomy, 31 Lenin Ave., 677980 Yakutsk, Russia
} 
To describe the observed properties of nonthermal emission of SN 1987A we use here a nonlinear kinetic theory. It couples the particle acceleration process with the hydrodynamics of the thermal gas (Berezhko et al. 1996; Berezhko \& Völk 2000). Therefore in spherically symmetric approach it is able to quite definitely predict the evolution of gas density, pressure, mass velocity, together with the energy spectrum and the spatial distribution of cosmic ray (CR) nuclei and electrons at any given evolutionary epoch $t$, and the properties of the nonthermal radiation produced in SNRs due to these accelerated CRs. The application of this theory to individual SNRs (see Berezhko 2005, for a review) has provided the explanation of the observed SNR properties under the assumption of strong interior magnetic field. It was also shown that this strong field leads to the concentration of the highest-energy electrons in a very thin shell just behind the shock. Recent observations with the Chandra and XMMNewton X-ray telescopes in space make it possible to resolve such filamentary structures, which are the result of strong synchrotron losses of the emitting multi-TeV electrons in strong magnetic fields downstream of the outer SN shock (Berezhko 2005).

Application of the nonlinear theory to SN 1987A (Berezhko \& Ksenofontov 2000) explained the properties of the observed radioemission. It predicted also the continuous increase of radioemission with roughly constant rate during the period from 1999 to $2006 \mathrm{yr}$, which was confirmed by further measurements (Manchester et al. 2002).

We analyze here radio data for more extended period of observation (Manchester \& Gaensler 2001; Manchester et al. 2002) together with the Chandra measurements of Xray emission (Park et al. 2004) which provides a strong constraint on the amount of the nonthermal synchrotron emission at the same energies $0.5-10 \mathrm{keV}$. It is demonstrated that this constrain gives the evidence of strongly enhanced (amplified) magnetic field inside the SNR, that in turn provides a better fit of radio data than in our previous study.

\section{Physical parameters of SNR 1987A}

We use canonical values of stellar ejecta mass $M_{e j}=10 M_{\odot}$, distance $d=50 \mathrm{kpc}$, hydrodynamic explosion energy $E_{s n}=1.5 \times 10^{51} \mathrm{erg}$ (e.g. McCray 1993). During an initial period the shell material has a broad distribution in velocity $v$. The fastest part of this ejecta distribution can be described by a power law $d M_{e j} / d v \propto v^{2-k}$. We use a value $k=8.6$ appropriate for SN 1987A (McCray 1993). The interaction of the ejecta with the circumstellar medium (CSM) creates a strong shock there which heats the thermal gas and accelerates particles diffusively to a nonthermal CR component of comparable energy density.

Strongly nonuniform CSM is a result of interaction of progenitors winds: dense slow RSG 
wind and subsequent fast and diluted BSG wind. During initial period after the explosion the SN shock propagated in the free BSG wind and reached after about a day $\sim 1000$ the termination shock in BSG wind, situated at the radial distance $R_{T}=3.1 \times 10^{17} \mathrm{~cm}$ (Berezhko \& Ksenofontov 2000). After that the SN shock propagates in the thermalized BSG wind of density $\rho_{B}$. Considerable rapid decrease of the SN shock speed occurred during the days $1500-2500$ (Gaensler et al. 1997) showed that SN shock entered the H II region of density $\rho_{R} \gg \rho_{B}$. Therefore we model the CSM density distribution at distances $r>R_{T}$ in the form

$$
\rho_{0}=\frac{\rho_{B}+\rho_{R}}{2}-\frac{\rho_{B}-\rho_{R}}{2} \tanh \frac{r-R_{C}}{l_{C}}
$$

where $R_{C}=6.1 \times 10^{17} \mathrm{~cm}$ is the contact discontinuity between two winds, $l_{C}=0.05 R_{C}$ is the scale of the smooth transition between them.

The analysis of the rapidly increasing X-ray emission provides the evidence that H II region in turn is not uniform: at day $\sim 6200$ the $\mathrm{SN}$ shock begins to interact with the dense inner ring. Its radial behavior can be represented in the form

$$
\rho_{R}=\frac{\rho_{R 1}+\rho_{R 2}}{2}-\frac{\rho_{R 1}-\rho_{R 2}}{2} \tanh \frac{r-R_{R}}{l_{R}},
$$

where $R_{R}=6.8 \times 10^{17} \mathrm{~cm}, l_{R}=0.08 R_{R}, \rho_{R 2} \approx 20 \rho_{R 1}$. Such a gas radial profile is close to what was extracted from the above analysis (Park et al. 2004). We adopt here for the gas number density $N_{g}=\rho / m_{p}$ the following values: $N_{g}^{B}=0.29 \mathrm{~cm}^{-3}, N_{g}^{R 1}=280 \mathrm{~cm}^{-3}$ and $N_{g}^{R 2}=4000 \mathrm{~cm}^{-3}$. Here $m_{p}$ is the mass of proton. The value $N_{g}^{R 1}=280 \mathrm{~cm}^{-3}$ which is by a factor of 1.5 lower than was used in our previous study (Berezhko \& Ksenofontov 2000), provides a good compromise between the SN shock dynamics seen in radio and in X-ray emissions (see below). Note, that the actual structure of the H II region, especially the dense inner ring, is essentially nonspherically symmetric. Nevertheless since our spherically symmetric theory reproduces the SN shock evolution $R_{s}(t)$ consistent with the existing data, one can expect that it gives a good estimate for the production of CRs and their subsequent nonthermal emission.

A rather high downstream magnetic field strength $B_{d} \sim 1 \mathrm{mG}$ is needed to reproduce the observed steep radio spectrum (Berezhko \& Ksenofontov 2000). We believe that the required strength of the magnetic field have to be attributed to nonlinear field amplification at the SN shock by CR acceleration itself. According to plasma physical considerations (Lucek \& Bell 2000; Bell 2004), the existing CSM magnetic field can indeed be significantly amplified at a strong shock by CR streaming instabilities. In fact, for all the thoroughly studied young SNRs, the ratio of magnetic field energy density $B_{0}^{2} / 8 \pi$ in the upstream region of the shock precursor to the CR pressure $P_{c}$ is about the same (Völk et al. 2005). Here $B_{0}=B_{\mathrm{d}} / \sigma$ is 
the far upstream field presumably amplified by CRs of highest energy, $\sigma$ is the total shock compression ratio. Within an error of about 50 percent we have $B_{0}^{2} /\left(8 \pi P_{c}\right) \approx 5 \times 10^{-3}$. CR pressure in young SNRs has a typical value $P_{c} \approx 0.5 \rho_{0} V_{s}^{2}$, therefore we adopt here upstream magnetic field

$$
B_{0}=\sqrt{2 \pi \times 10^{-3} \rho_{0} V_{s}^{2}}
$$

Since the process of magnetic field amplification is not included in our theory we simply postulate the existence of far upstream field $B_{0}$ given by Eq.(3). The spectrum of CRs produced by strong modified shock is very hard so that CRs with highest energies have a largest contribution in their energy content. These the most energetic CRs produce field amplification on their spacial scale that is the precursor size. Therefore CRs with lower energies already 'see' the amplified field $B_{0}$. We will argue that such a high field, that is by a factor of ten larger than used in our previous study (Berezhko \& Ksenofontov 2000), is indeed required to fit the X-ray data. It also gives a better fit of radio data.

We start our consideration from the SNR evolutionary epoch $t=1000 \mathrm{~d}$, when the outer SN shock has a radius $R_{i}=R_{T}$ and speed $V_{i}=28000 \mathrm{~km} / \mathrm{s}$. These values of $R_{s}$ and $V_{s}$ according to our calculations (Berezhko \& Ksenofontov 2000) correspond to the end of SN shock propagation in the free BSG wind region $r<R_{T}$. We neglect the contribution of CRs accelerated in the region $r<R_{T}$, because due to a high gas density the number of CRs produced in the region $r>R_{T}$ very soon becomes dominant.

\section{Results and Discussion}

Calculated shock radius $R_{s}$ and speed $V_{s}$ shown in Fig.1a as a function of time are in satisfactory agreement with the values obtained on the basis of radio and X-ray measurements. Note that radio data compared with X-ray data gives larger shock size at any given time $t$ and calculated radius $R_{s}(t)$ goes between these two sets

To fit the spectral shape of the observed radio emission we assume a proton injection rate $\eta=3 \times 10^{-3}$, which is a fraction of gas particles involved into the acceleration at the from of SN shock. This leads to a significant nonlinear modification of the shock: as it is seen in Fig.1b total shock compression ratio $\sigma \approx 5.3$ is essentially larger and a subshock compression ratio $\sigma_{s} \approx 2.8$ is lower than classical value 4 .

Since the SN 1987A is very young CRs despite of very efficient acceleration accumulated only $7 \%$ of the explosion energy $E_{s n}$ (see Fig.1c). Note that the injection is expected to be strongly suppressed at the quasiperpendicular part of the shock, therefore one should renormalize the results for the nucleonic spectrum, calculated within the spherically symmetric 
model. The lack of symmetry in the actual SNR can be approximately taken into account by a renormalization factor $f_{r e} \approx 0.2$, which diminishes the nucleonic CR production efficiency, calculated in the spherical model, and all effects associated with it (see Völk et al. 2003, for details). With this renormalization the CRs inside SN 1987A already contain

$$
E_{c}=0.07 f_{r e} E_{s n} \approx 2 \times 10^{49} \mathrm{erg} .
$$

Since during the last 15 yrs the shock speed and the gas density are almost constant CR energy content grows roughly linearly with time. It gives the natural explanation of the linear increase of the radio emission detected during this evolutionary period.

Strongly modified SN shock generates $\mathrm{CR}$ spectrum $N \propto p^{-\gamma}$, which is very steep at momenta $p<m_{p} c$, with index $\gamma=\left(\sigma_{s}+2\right) /\left(\sigma_{s}-1\right) \approx 2.7$. CR electrons with such a spectrum produces synchrotron radioemission spectrum $S_{\nu} \propto \nu^{-\alpha}$ with spectral index $\alpha=(\gamma-1) / 2 \approx 0.9$, that very well corresponds to the experiment, as it is seen in Fig.2, where we present synchrotron energy spectra $\nu S_{\nu}$, calculated for five subsequent epoch together with the experimental data. Note that CR spectrum has a concave shape: it becomes flatter at higher momenta $p$. As a consequence synchrotron spectrum $S_{\nu}(\nu)$ is also concave as it is clearly seen in Fig. 2 at $\nu<10^{12} \mathrm{~Hz}$. Radio data reveal this property in good consistency with theoretical prediction.

Strong downstream magnetic field $B_{d} \approx 15 \mathrm{mG}$, that corresponds to the upstream field $B_{0} \approx 3 \mathrm{mG}$ (see Fig.1), provides synchrotron cooling of electrons with momenta $p>10 m_{p} c$ that in turn makes synchrotron spectrum at high frequencies $\nu>10^{12} \mathrm{~Hz}$ very steep (see Fig.2). Concave shape of electrons continuously produced at the shock front together with their synchrotron cooling lead to a formation of two peaks in synchrotron energy spectrum $\nu S_{\nu}$. The first one at $\nu \approx 10^{12} \mathrm{~Hz}$ corresponds to $\mathrm{CR}$ electron momentum $p \approx 10 m_{p} c$ above which synchrotron energy looses are relevant, whereas the second peak at $\nu \approx 10^{18} \mathrm{~Hz}$ corresponds to the maximum momentum $p \approx 10^{4} m_{p} c$ of accelerated electrons. Under this condition calculated synchrotron flux at frequency $\nu \approx 10^{17} \mathrm{~Hz}$, which corresponds to the photon energy $\epsilon_{\gamma}=0.5 \mathrm{keV}$, is below the measured flux at the epochs $t>3000 \mathrm{~d}$. Since the contribution of the nonthermal radiation in the observed X-ray emission of SN 1987A is not very well known (e.g. Michael et al. 2002), the observed X-ray flux has to be considered as the upper limit for the expected nonthermal emission. At early epoch $t<2500 \mathrm{~d}$ however the calculated flux exceeds the measured one (see the curve, corresponding $t=1970 \mathrm{~d}$ in Fig.2). This can be considered as indication, that the actual magnetic field $B_{0}$ is few times larger then given by the Eq.(3).

To illustrate the situation expected at considerably lower magnetic field we present in Fig.3 synchrotron energy spectra calculated at the same set of parameters as before except 
magnetic field, which was taken $B_{0}=200 \mu \mathrm{G}$ independent of time. Since the interior magnetic field $B_{d} \approx 2 \mathrm{mG}(\sigma \approx 10)$ is essentially lower in this case, synchrotron losses of high energy $\mathrm{CR}$ electrons are considerably smaller compared with the previous case. Due to this fact synchrotron spectra considerably exceeds at any given epoch the measured Chandra flux. Therefore we can conclude, that the actual interior magnetic field strength is not lower than $5 \mathrm{mG}$.

Projected radial profile of the nonthermal X-ray emission at high interior magnetic field $B_{d}=10 \mathrm{mG}$ is characterized by an extremely sharp peak of thickness $L \approx 10^{-3} R_{s}$ just behind the SN shock. Since the thermal X-rays have much wider radial distribution it provides the possibility for experimental determination of the nonthermal X-ray contribution in the observed emission. Experimental determination of the X-ray spectrum at $\nu=10^{18}-10^{19} \mathrm{~Hz}$ $\left(\epsilon_{\gamma}=5-50 \mathrm{keV}\right)$ is the another possibility to discriminate the nonthermal emission. Such kind of measurements can be done with space instrument Suzaku.

Completely different possibility to have the synchrotron flux, which fit the radio data and goes below Chandra data, is low field scenario, when the magnetic field is as low as $B_{0}<2 \mu \mathrm{G}$. In such a case the cutoff frequency of synchrotron spectrum $\nu_{\max }$ is lower than $10^{17} \mathrm{~Hz}$ and calculated fluxes go down exponentially at $\nu>\nu_{\max }$ below Chandra points. However the value $B_{0}<2 \mu \mathrm{G}$ is unrealistically small for such a dense medium, which we have in SN 1987A. In addition the effective energy of electrons

$$
\epsilon_{e} \approx 5 \sqrt{(\nu / 1 \mathrm{GHz}) /\left(B_{d} / 10 \mu \mathrm{G}\right)} \mathrm{GeV}
$$

which radiate at $\nu \sim 1 \mathrm{GHz}$ in the interior field $B_{d}=10 \mu \mathrm{G}$ is about $5 \mathrm{GeV}$. The electron spectrum at $\mathrm{GeV}$-energies is characterized by a power low index $\gamma \approx 2$ that is considerably smaller than what is required for the observed radio spectrum. Note that as it is seen in Fig.3 even the field $B_{d} \approx 2 \mathrm{mG}$ is too small to have a good fit of radio data as in Fig.2. Therefore low field scenario should be rejected.

Calculated $\gamma$-ray integral flux shown in Fig.4 at all energies is dominated by the $\pi^{0}$-decay component. Since the SN shock is strongly modified $\gamma$-ray spectrum at energies $\epsilon_{\gamma}>0.1 \mathrm{TeV}$ is very hard: $F_{\gamma} \propto \epsilon_{\gamma}^{-0.9}$. At the current epoch the expected $\gamma$-ray energy flux at TeV-energies is about $\epsilon_{\gamma} F_{\gamma} \approx 2 \times 10^{-13} \mathrm{erg} /\left(\mathrm{cm}^{2} \mathrm{~s}\right)$ and during the next four years it expect to grow by a factor of two. This flux is by a factor of five lower compared with our previous prediction (Berezhko \& Ksenofontov 2000), because we use here renormalization factor $f_{r e}=0.2$, which reduces the number of accelerated CRs calculated within spherically symmetric approach. The existence of strongly asymmetric CSM structure, which is dense inner ring, makes our prediction of $\gamma$-ray flux uncertain. According to the rough estimate this uncertainty is not very large, about a factor of two, due to the stronger SN shock deceleration in denser medium. 
At the moment there are only upper limits of $\mathrm{TeV}$ emission obtained by CANGAROO (Enomoto et al. 2003) and HESS (Rowell et al. 2004) instruments (see Fig.4).

\section{Summary}

The kinetic nonlinear model for CR acceleration in SNRs has been in detail applied to SN 1987A, in order to compare its results with observational properties. We find that quite a reasonable consistency with most of the observational data can be achieved.

The evidence of the efficient CR production leading to a strong shock modification comes from radio data. Significant shock modification leads to the steep and concave CR spectrum which very well fits the observed radioemission spectrum, under the condition of extremely high downstream magnetic field strength $B_{d} \sim 1 \mathrm{mG}$. To be consistent also with the Chandra measurements of the X-ray flux, which has to be considered as an upper limit for the nonthermal X-ray emission, even larger interior field $B_{d} \approx 10 \mathrm{mG}$ is needed. Such a high field provides a strong synchrotron losses of CR electrons emitting nonthermal X-rays, that makes the high frequency part of the synchrotron spectrum much more steeper and provides a consistency with the experiment.

The expected $\pi^{0}$-decay $\gamma$-ray energy flux at the current epoch is about $\epsilon_{\gamma} F_{\gamma} \approx 2 \times 10^{-13}$ $\mathrm{erg} /\left(\mathrm{cm}^{2} \mathrm{~s}\right)$ at energies $\epsilon_{\gamma}=0.1-10 \mathrm{TeV}$. Therefore the detection of $\gamma$-ray emission at these energies would imply clear evidence for a hadronic origin and for a strong magnetic field amplification inside SN 1987A.

\section{REFERENCES}

Bell, A. R. 2004, MNRAS, 353, 550

Berezhko, E. G., Elshin, V. K., \& Ksenofontov, L. T. 1996, JETP, 82, 1

Berezhko, E.G. \& Völk, H.J. 2000, A\&A, 357, 183

Berezhko, E. G., \& Ksenofontov, L. T. 2000, Astronomy Letters, 26, 639

Berezhko, E.G. 2005, Adv. Space Res., 35, 1031

Chevalier, R. A. \& Dwarkadas, V. V. 1995, ApJ, 452, L45

Chevalier, R. A., \& Fransson, C. 1987, Nature, 328, 44 
Enomoto, R. et al. 2003, ApJ, 591, L25

Gaensler, B. M. et al. 1997, ApJ, 479, 845

Gorenstein, P., Hughes, J. P., Tucker, W. H. 1994, ApJ, 420, L25

Hasinger, G., Ashenbach, B. \& Trümper, J. 1996, A\&A, 312, L9

Lucek, S. G. \& Bell, A. R. 2000, MNRAS, 314, 65

McCray, R. 1993, ARA\&A, 31175

Manchester, R. N. et al. 2002, PASA, 19, 207

Manchester, R. N. \& Gaensler, B. M. 2001, IAU Circ. 7757

Michael, E. et al. 2002, ApJ, 574, 166

Park, S. et al. 2004, ApJ, 610, 275

Rowell, G. et al. 2004, AIP Conf. Proc., 745, 299

Staveley-Smith, L., et al. 1992, Nature, 355, 147

Turtle, A. J. et al. 1987, Nature, 327, 38

Völk, H. J., Berezhko, E. G., \& Ksenofontov, L. T. 2003, A\&A409, 563

Völk, H. J., Berezhko, E. G., \& Ksenofontov, L. T. 2005, A\&A, 433, 229. 


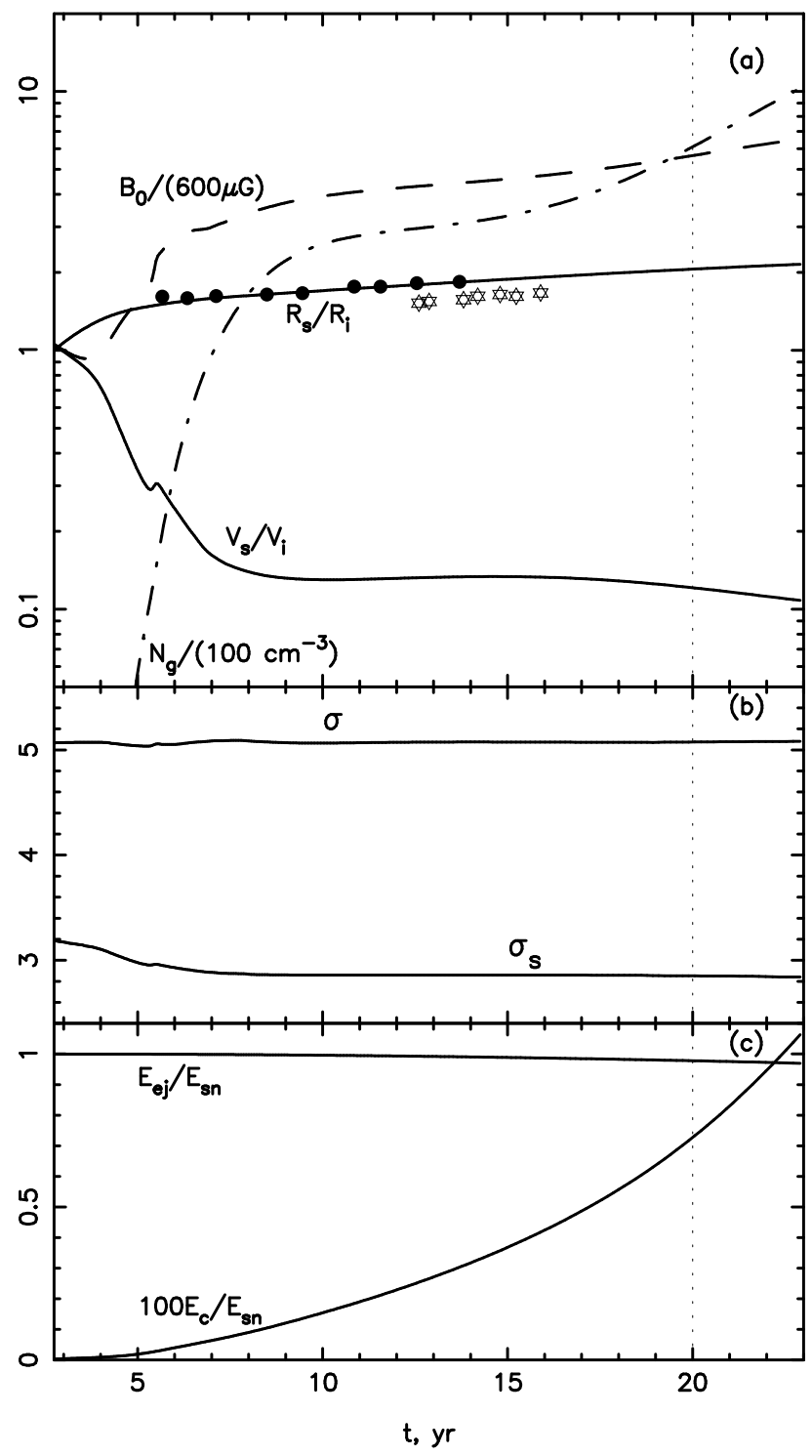

Fig. 1.- (a) Shock radius $R_{\mathrm{s}}$, shock speed $V_{\mathrm{s}}$, gas density $N_{g}$ and upstream magnetic field $B_{0}$ at the current shock position; (b) total shock $(\sigma)$ and subshock $\left(\sigma_{\mathrm{s}}\right)$ compression ratios; (c) kinetic energy of ejecta $E_{e j}$ and accelerated CR energy content $E_{c}$ as functions of time. The dotted vertical line marks the current epoch. The observed radius of the SN shock, as determined by radio (Manchester et al. 2002) and X-ray measurements (Park et al. 2004), are shown by circles and stars respectively. The scaling values are $R_{i}=R_{T}=3.1 \times 10^{17} \mathrm{~cm}$ and $V_{i}=28000 \mathrm{~km} / \mathrm{s}$ 


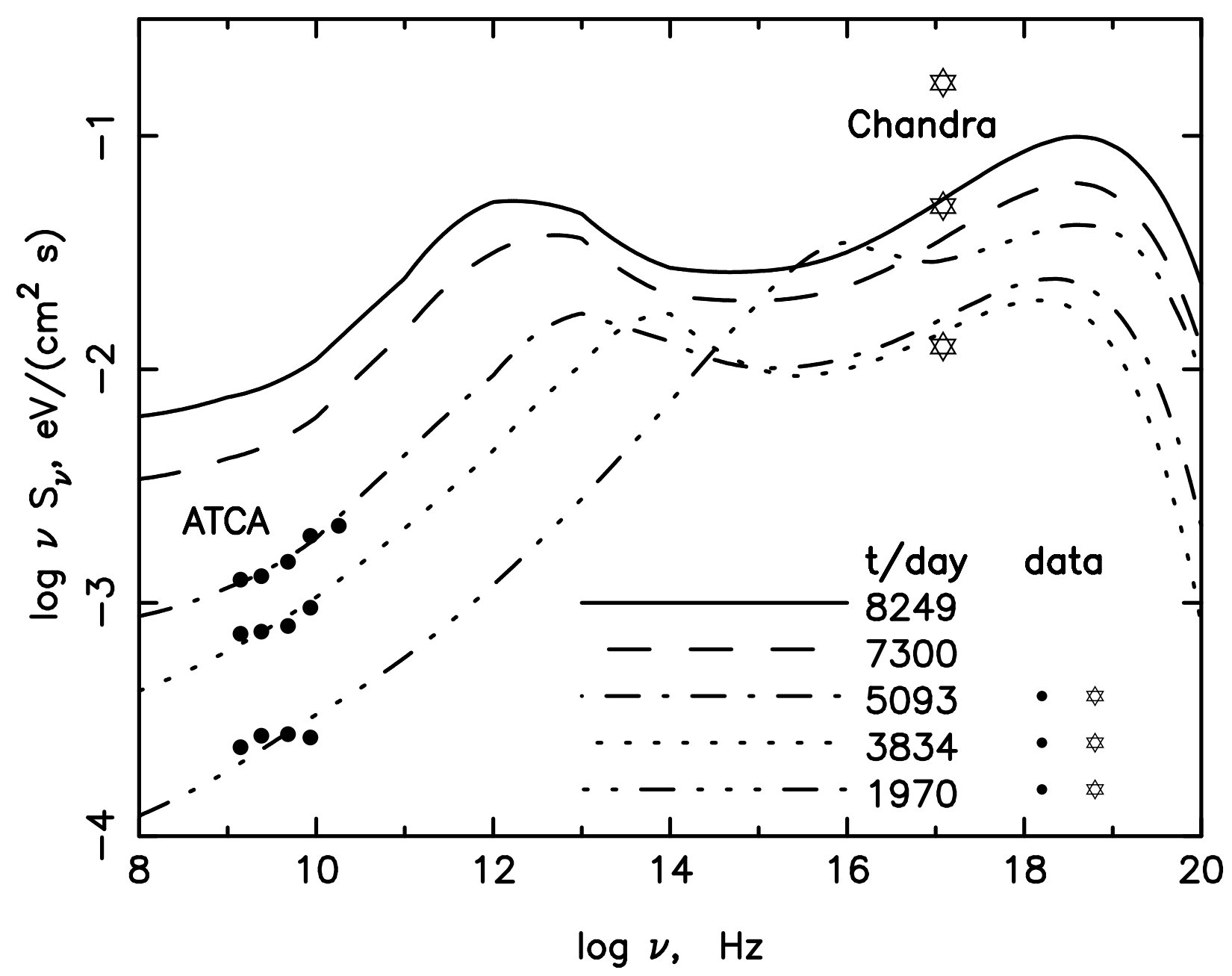

Fig. 2.- Synchrotron energy spectrum of SN 1987A, calculated for the five evolutionary epochs. The ATCA radio (Manchester et al. 2002; Manchester \& Gaensler 2001) and Chandra X-ray (Park et al. 2004) data for three epochs are shown as well. Higher measured fluxes correspond to later epoch. 


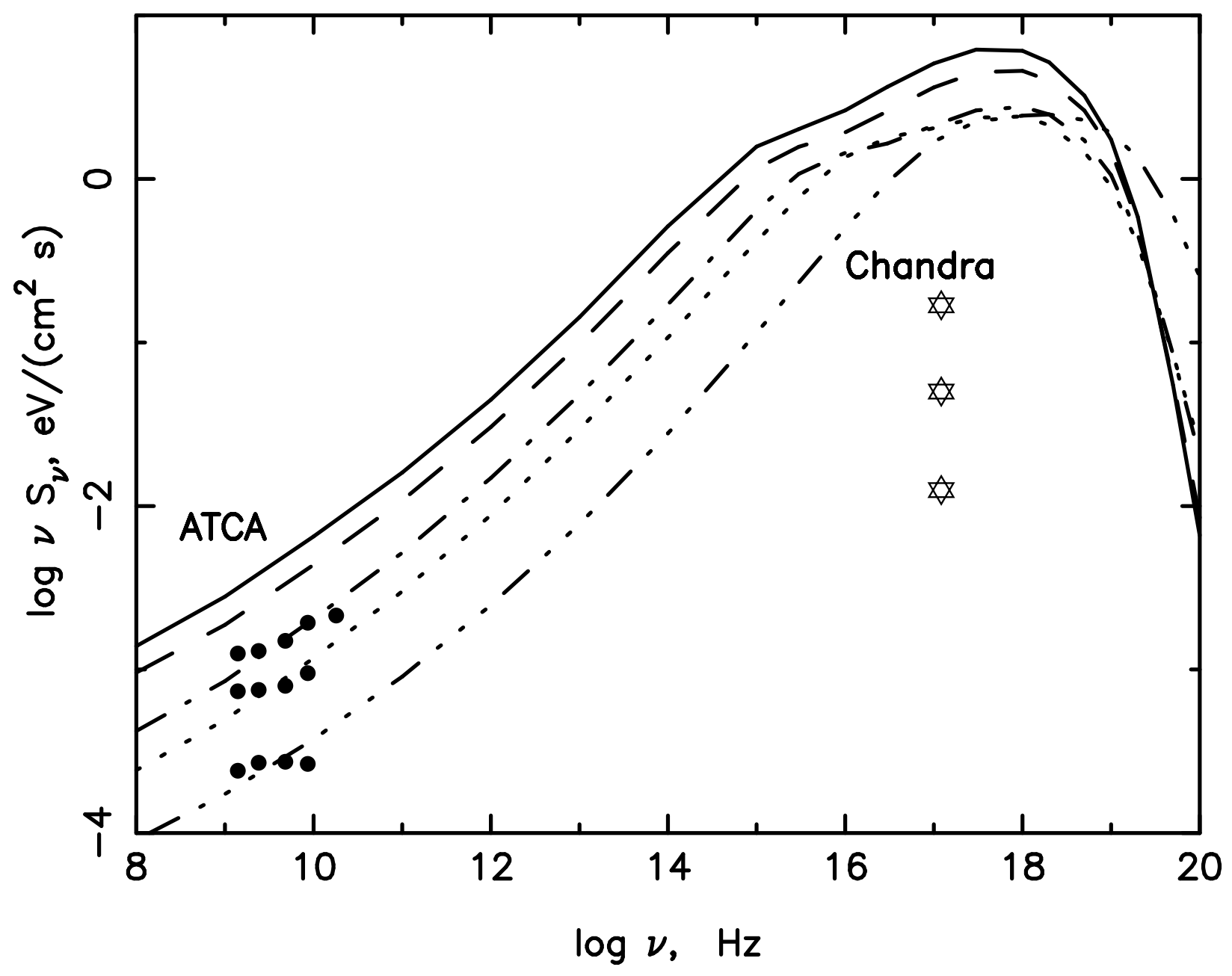

Fig. 3.- Same as in Fig.2, but the calculations are for the case of constant upstream magnetic field $B_{0}=200 \mu \mathrm{G}$. 


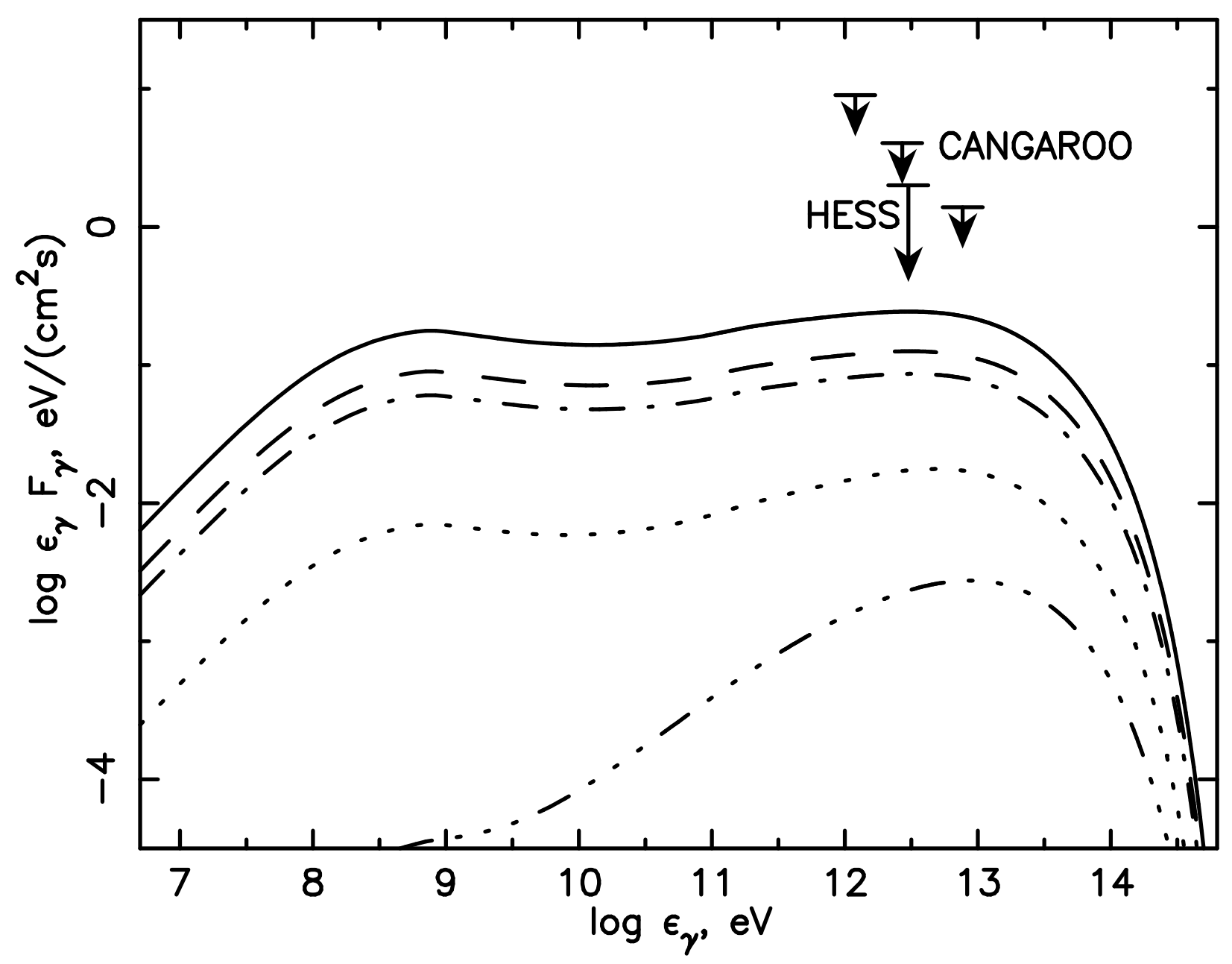

Fig. 4.- Integral $\gamma$-ray energy flux from SN 1987A, calculated for the same five epoch as in Fig.2. CANGAROO (Enomoto et al. 2003) and HESS (Rowell et al. 2004) upper limits are shown as well. 